Meta

Journal des traducteurs

Translators' Journal

\title{
Les institutions de traducteurs et d'interprètes chinois, leurs activités et leurs publications
}

\section{She Xiebin}

Volume 44, numéro 1, mars 1999

Théorie et pratique de la traduction en Chine

The Theory and Practice of Translation in China

URI : https://id.erudit.org/iderudit/004130ar

DOI : https://doi.org/10.7202/004130ar

Aller au sommaire du numéro

Éditeur(s)

Les Presses de l'Université de Montréal

ISSN

0026-0452 (imprimé)

1492-1421 (numérique)

Découvrir la revue

Citer cet article

Xiebin, S. (1999). Les institutions de traducteurs et d'interprètes chinois, leurs activités et leurs publications. Meta, 44(1), 209-213.

https://doi.org/10.7202/004130ar
Résumé de l'article

La Chine est le pays qui compte le plus grand nombre de traducteurs. Depuis l'instauration de sa politique d'ouverture et de réforme, le pays aborde une nouvelle étape de développement général dans ce domaine. Combien la Chine compte-t-elle de traducteurs ? Quelles sociétés savantes de traducteurs y ont été fondées ? Quelles activités universitaires ont été créées ? Quelles sont les revues savantes et les autres publications dans le domaine ? Cet article veut répondre à ces questions spécialement à l'intention des traducteurs des autres pays. 


\title{
Les institutions de traducteurs et d'interprètes chinois, leurs activités et leurs publications
}

\author{
she xiebin \\ Université des Chemins de Fer de Changsha, \\ Changsha, Chine
}

\begin{abstract}
RÉSUMÉ
La Chine est le pays qui compte le plus grand nombre de traducteurs. Depuis l'instauration de sa politique d'ouverture et de réforme, le pays aborde une nouvelle étape de développement général dans ce domaine. Combien la Chine compte-t-elle de traducteurs? Quelles sociétés savantes de traducteurs y ont été fondées? Quelles activités universitaires ont été créées? Quelles sont les revues savantes et les autres publications dans le domaine? Cet article veut répondre à ces questions spécialement à l'intention des traducteurs des autres pays.
\end{abstract}

\section{ABSTRACT}

China has more translators than any other countries in the world. Since the national policy of reform and opening was carried out, China has entered a new stage of all-round development in the cause of translating. Then, how many translators does China have? What learned societies of translation have been set up? What academic activities have been carried out? What academic journals and works have been published? This paper tries to answer those questions people of the same trade abroad are interested in.

La traduction chinoise a une histoire de près de 2000 ans au cours de laquelle elle a connu trois essors: celui de la traduction des ouvrages canoniques du bouddhisme à partir de la dynastie des Han de l'Est (25-220 avant J.-C.) jusqu'à la dynastie des Song (960-1127) ; celui de la traduction d'ouvrages scientifiques et techniques à partir de la fin de la dynastie des Ming (1368-1644) jusqu'au début de la dynastie des Qing (1644-1911) ; celui de la traduction des connaissances de l'O ccident après la guerre de I'O pium (1840) jusqu'à la fondation de la République populaire de Chine (1949). Les traducteurs des époques passées ont apporté, par leur travail inlassable et créatif, de grandes contributions au développement de la société chinoise et au progrès de l'humanité.

A près la fondation de la Chine nouvelle, et surtout après la grande catastrophe de la Révolution culturelle (1966-1976) et I'application de la politique de réforme et d'ouverture sur l'extérieur, la Chine est entrée dans une époque prospère pour la traduction dans tous les domaines. Selon les statistiques, on compte actuellement en Chine environ un million de traducteurs et d'interprètes (professionnels ou amateurs) dispersés dans les organismes du gouvernement, qui traduisent dans les domaines des sciences et des techniques, de la littérature et de l'art, de la culture et de l'éducation, de l'économie et du commerce, etc. Ils forment un contingent important de l'armée des traducteurs du monde. 
Cet article a pour but de présenter brièvement les institutions de traducteurs chinois, ainsi que leurs activités et leurs publications, pour donner à nos collègues étrangers un aperçu général sur les milieux de la traduction chinoise.

\section{INSTITUTIONS DE TRADUCTEURS ET INTERPRÈTES CHINOIS}

Dans la Chine antique, on voyait déjà des institutions ou des organismes de traduction: ainsi, par exemple, on a organisé des cours de traduction des ouvrages canoniques du bouddhisme sous les dynasties des Tang et des Song. Et des organismes de traduction de toutes sortes ont été fondés successivement après la guerre de l'O pium, dont certains dirigés par des missionnaires étrangers, tels que The London Mission Press, créé en 1843 par Dr Water Henry M odhurst; Tenchow College, créé en 1864 par Calvin W. Mateer ; The School and Text Book Series Committee, créé en 1877 par John Fryer; la Society for the Diffusion of Christian and General Knowledge Among the Chinese, créé en 1887 par A. Williamson, etc.

Après la libération, en 1949, et avec l'ouverture du pays, les traducteurs et interprètes sont devenus de plus en plus nombreux. Pour mieux répondre aux besoins du développement de la cause de la traduction, ils ont fondé leurs propres institutions à divers échelons.

Au niveau national, I'Association des traducteurs de Chine (The Translators' Association of China) a été fondée en 1982; elle comprend, à I'heure actuelle, 7 comités spécifiques:

- le Comité de traduction des sciences sociales;

- le Comité de traduction de la littérature et de l'art;

- le Comité de traduction des sciences et des techniques;

- le Comité de traduction des langues des minorités nationales chinoises;

- le Comité de traduction de la diplomatie, de la presse et de la diffusion à l'étranger;

- le Comité de la théorie et de l'enseignement de la traduction.

Au niveau régional, on compte jusqu'à présent au moins 47 associations de traducteurs de provinces, de régions autonomes et de municipalités autonomes comprenant plus de 30000 membres, sans compter ceux des associations de traducteurs d'arrondissements, de villes, de districts, d'établissements et d'universités. Toutes ces institutions régionales font partie de l'Association des traducteurs de Chine, dirigée par un conseil national composé actuellement de 242 membres dont le président est le célèbre traducteur Ye Shuifu.

On distingue aussi, parmi ces institutions à différents échelons, des associations spécifiques telles que:

- I'Association des traducteurs littéraires;

- I'Association des traducteurs scientifiques et techniques;

- I'Association des interprètes;

- I'Association des traducteurs d'entreprise;

- I'Association des jeunes traducteurs;

- l'Association des aspirants-chercheurs pour la traduction; etc.

Toutes les institutions de traducteurs chinois ont leurs statuts stipulant les droits et les intérêts légitimes ainsi que les obligations de leurs membres. 


\section{LES ACTIVITÉS DES INSTITUTIONS DE TRADUCTEURS CHINOIS}

Les institutions de traducteurs chinois sont des sociétés académiques de masse dont le but principal est d'unir et d'organiser les traducteurs pour développer les études et les échanges de traduction, ainsi que d'élever la qualité professionnelle. À cet effet, on entreprend des activités de toutes sortes:

- Organisation de colloques de traduction: de 1982 à 1992, 23 colloques au niveau national ont été organisés soit sur la traduction des ouvrages littéraires ou techno-scientifiques, soit sur la traduction écrite ou l'interprétation, soit sur la théorie ou l'enseignement de la traduction ; 2200 personnes ont participé à ces rencontres où environ 1500 communications ont été présentées. En même temps, les associations régionales ont organisé, elles aussi, 534 colloques sur la traduction dans tout le pays.

- Formation de jeunes traducteurs ou interprètes par des conférences, séminaires ou concours de traduction. Selon des statistiques incomplètes, de 1982 à 1992, les institutions de traducteurs chinois ont organisé 279 conférences et 820 séminaires ou stages; plus de 50000 jeunes «cadres de traduction » ont été formés. L'Institut de formation des traducteurs et des interprètes, fondé par l'Association des traducteurs de Xian (chef-lieu de la province du Shanxi) en est un bon exemple. De plus, on a organisé jusqu'à présent 11 concours nationaux pour les étudiants et les jeunes professeurs et traducteurs, dont la plupart sont sous le patronage de $\mathrm{M}{ }^{\mathrm{me}} \mathrm{H}$ an Suyin, célèbre écrivain chinois de nationalité anglaise.

- Organisation de grands travaux de traduction pour le progrès social et le développement de la production. Prenons pour exemple la traduction littéraire: de 1980 à 1989, on a traduit plus de 60000 ouvrages littéraires de près de 2000 écrivains étrangers. Les œuvres complètes de Balzac, de Shakespeare, de Tolstoï, de Gorki... ont déjà vu le jour en chinois. En outre, la Collection de la littérature de Panda et beaucoup d'autres publications présentent sans cesse la littérature moderne, contemporaine et classique chinoise aux lecteurs étrangers.

Ne parlons pas ici des tonnes et des tonnes de documents scientifiques et techniques traduits en chinois. Jetons un coup d'œil sur la traduction en langues des minorités nationales qui occupe une place particulière étant donné les 55 nationalités minoritaires que comprend la Chine. En 1990, par exemple, on a publié 3255 livres différents comprenant 38670000 exemplaires en langues des minorités nationales, 131 magazines ou revues différents dont le tirage a atteint 3130000 exemplaires, ainsi que 77 journaux différents en 148350000 exemplaires; une bonne partie de ces publications sont des traductions.

- Développement des recherches scientifiques en traduction. À partir de 1989, de nombreux projets de recherche sur la traduction ont été établis et soutenus, soit par les fonds nationaux pour les études de la philosophie et des sciences sociales, soit par les fonds de la Commission de l'éducation nationale pour les études des sciences humaines, projets tels que:

Absorption des idées politiques et philosophiques occidentales par la Chine et le Japon contemporains à travers la traduction, 1989, présidé par Wang Kefei;

Histoire de la traduction des territoires de l'Ouest (sous les $\mathrm{Han}$, les régions à l'ouest de Yumenguan au Gansu, comprenant le Xinjiang d'aujourd'hui et une partie de l'Asie centrale), 1989, présidé par Rezake M aitiniyazi ; 
Études de la théorie de traduction, 1990, présidé d'abord par Chen Zongbao puis par Xu Jun ; Généralités sur la traduction des littératures anglaise et américaine (1949-1966), 1993, présidé

par Sum Zhili ;

La traduction et la sémantique comparée, 1949, présidé par Wang Bingqin ;

Précis de l'histoire de la traduction littéraire, 1996, présidé par Xie Tiangzen;

Études des problèmes fondamentaux de la traduction littéraire, 1996, présidé par Xu Jun ;

Études de l'enseignement de traduction, 1996, présidé par Mu Lei, etc.

- Développement des discussions et des critiques sur les ouvrages traduits. C'est souvent difficile à faire, mais ces dernières années ce nouveau genre est apparu grâce, en particulier, à la discussion de la retraduction d'un chef-d'œuvre de Stendhal Le Rouge et le Noir en 1995 et 1996. Xu Jun, professeur titulaire de I'Université de Nanjing (Nankin) et traducteur d'élite, a enregistré fidèlement cette discussion dans sa monographie intitulée Écriture, Littérature et Culture: études des traductions du Rouge et le Noir, qui est un document très important concernant la critique de la traduction.

- Renforcement des liens et des échanges avec les milieux des traducteurs des pays étrangers. En 1987, I'Association des traducteurs de Chine est devenue officiellement membre de la Fédération internationale des traducteurs. Dès lors, elle a envoyé plusieurs délégations aux congrès de la FIT et effectué des visites de travail dans les pays étrangers. Elle a invité aussi des collègues étrangers comme Eugene A. Nida et Danica Seleskovitch à venir en Chine faire des conférences. Ce qui renforce davantage la connaissance et l'amitié entre les traducteurs chinois et étrangers.

\section{LES PUBLICATIONS DES INSTITUTIONS DE TRADUCTEURS CHINOIS}

Les associations nationales et une bonne partie des associations régionales ont leurs actes ou bulletins académiques dans lesquels leurs membres peuvent publier des articles ou des essais sur la traduction. Leur but étant:

- de développer les études de la théorie de la traduction;

- d'échanger les expériences de traduction;

- de commenter des ouvrages traduits;

- de diffuser les connaissances sur la traduction;

- de promouvoir l'enseignement de la traduction;

- de présenter des traducteurs d'élite;

- de rapporter des courants d'idées et l'évolution de la traduction de l'intérieur et de l'extérieur du pays.

Les actes les plus connus sont les suivants:

- Journal des traducteurs chinois, actes académiques de l'Association des traducteurs de Chine (créé en 1983, près de 2000 articles y ont été publiés) ;

- Journal des traducteurs scientifiques et techniques chinois, actes académiques de l'Association des traducteurs scientifiques et techniques de I'Académie des sciences de Chine, créé en 1988;

- Ami des traducteurs;

- Langues et traduction;

- Études de traduction;

- Journal des traducteurs littéraires; 
- Journal des traducteurs scientifiques et techniques de Shanghai ;

- Journal des traducteurs des minorités nationales.

On publie aussi des articles concernant la traduction dans des revues académiques des universités, dont voici les plus connues:

- Enseignement et études des langues étrangères, revue académique de l'Université des études étrangères de Beijing (Pékin) ;

- Langues étrangères, revue académique de l'Université des études internationales de Shanghai ;

- Langues étrangères modernes, revue académique de l'Université des études étrangères de Guangdong;

- $\quad$ Langues étrangères et traduction, revue académique de I'U niversité des Chemins de fer de Changsha (Hunan), dont je suis le rédacteur en chef adjoint.

Outre plus de 6000 articles sur la traduction publiés dans divers journaux ou revues, plus de 300 ouvrages de traduction ont vu le jour à partir des années 1980; en voici quelques exemples:

Ma Zuyi, Précis de I'histoire de la traduction chinoise, 1984;

Tan Zaixi, Précis de l'histoire de la traduction occidentale, 1991;

Chen Yugang, Document historique de la littérature traduite chinoise, 1989;

Luo Xinzhang, Collection des essais de traduction, 1984;

Xu Yuanchong, Art de la traduction, 1984;

Zhang Jin, Principes de traduction littéraire, 1987;

Liu Zhongde, Dix discours sur la traduction littéraire, 1991;

Qian Gechuan, La Technique de traduction, 1981;

Chen Zhongbao, Cours de traduction du français au chinois, 1984;

Jin Di et Eugene A. Nida, De la traduction, 1984;

Zhang Peiji, Cours de traduction de l'anglais au chinois, 1983;

Xu Jun, Études de la critique de la traduction littéraire, 1992;

Xu Jun, Écriture, littérature et culture. Études des traductions chinoises du Rouge et le Noir, 1996;

Lin Hui, Dictionnaire des traducteurs chinois, 1988;

Lin Huangtian, Dictionnaire de la traduction chinoise, 1997, etc.

Pour que les lecteurs étrangers puissent connaître la politique, l'économie et la culture de la Chine, on édite aussi une série de magazines ou de revues illustrées en plusieurs langues étrangères telles que Littérature chinoise, La Chine au présent, Beijing Information, La Chine picturale, etc. 